\title{
Improved detectability of hypoattenuating focal pancreatic lesions by dual-layer computed tomography using virtual monoenergetic images
}

\author{
Nada El Kayal ${ }^{1,2^{*}}$ D, Ahmed Mohallel ${ }^{1}$, David Maintz ${ }^{2}$, Mohamed Eid ${ }^{1}$ and Doaa Mokhtar Emara ${ }^{1}$
}

\begin{abstract}
Background: Multidetector $C T$ is the mainstay for radiologic evaluation of pancreatic pathology. Still, imaging of focal pancreatic lesions using MDCT is faced by a number of challenges that are related to the limited contrast between the lesion and surrounding parenchyma, such as detecting early-stage pancreatic cancer and subtle features of cystic lesions that point to malignancy. Dual-layer $C T$ is the first dual-energy $C T$ machine based on separation of high- and low-energy photons at the detector level. If improved contrast between the lesions and normal pancreatic parenchyma could be achieved on $\mathrm{CT}$ images, we may expect enhanced $\mathrm{CT}$ detection of pancreatic lesions. The purpose of this study was to evaluate whether virtual monoenergetic reconstructions generated using contrast-enhanced dual-layer CT could improve detectability of hypoattenuating focal pancreatic lesions compared to conventional polyenergetic reconstructions.

Results: Fifty-four lesions were identified and verified by histopathology or follow-up CT, MRCP, and/or EUS along with clinical data. Across the virtual monoenergetic spectrum, $40 \mathrm{KeV}$ images had the highest contrast-to-noise and signal-to-noise ratios $(p<0.001, p<0.001)$ and were significantly higher than conventional images $(p<0.001)$. Subjective scores for lesion visibility at low kiloelectron volt monoenergetic (40 and $50 \mathrm{KeV}$ ) images greatly exceeded conventional images $(p<0.001)$.
\end{abstract}

Conclusion: Low kiloelectron volt monoenergetic reconstructions of contrast-enhanced dual-layer CT significantly improve detectability of hypoattenuating focal pancreatic lesions compared to conventional polyenergetic reconstructions.

Keywords: Dual-energy computed tomography, Dual-layer computed tomography, Pancreatic lesions, Pancreas, Pancreatic ductal adenocarcinoma

\footnotetext{
* Correspondence: nadaelkayal@hotmail.com

'Departmant of Diagnostic and Interventional Radiology, Faculty of Medicine, Alexandria University, Alexandria, Egypt

${ }^{2}$ Institute for Diagnostic and Interventional Radiology, University Hospital of Cologne, Cologne, Germany
}

\section{Springer Open}

(c) The Author(s). 2020 Open Access This article is licensed under a Creative Commons Attribution 4.0 International License, which permits use, sharing, adaptation, distribution and reproduction in any medium or format, as long as you give appropriate credit to the original author(s) and the source, provide a link to the Creative Commons licence, and indicate if changes were made. The images or other third party material in this article are included in the article's Creative Commons licence, unless indicated otherwise in a credit line to the material. If material is not included in the article's Creative Commons licence and your intended use is not permitted by statutory regulation or exceeds the permitted use, you will need to obtain permission directly from the copyright holder. To view a copy of this licence, visit http://creativecommons.org/licenses/by/4.0/. 


\section{Background}

Multidetector computed tomography (MDCT) is the mainstay for radiologic evaluation of pancreatic pathology [1]. Current pancreatic MDCT protocol comprises a multiphasic technique including non-contrast, pancreatic parenchymal (PPP) and portal venous (PVP) phases [2]. Pancreatic ductal adenocarcinoma is the most common among pancreatic malignancies (constituting 85-95\%) and continues to be a leading cause of cancer-deaths, carrying high mortality and poor prognosis [3-5]. Pancreatic adenocarcinoma typically appears hypoattenuating relative to surrounding pancreas parenchyma on MDCT and is best detected on pancreatic-phase. Comprehensive assessment of vascular invasion and distant metastases also necessitates portal venous phase imaging $[6,7]$.

Despite recent developments in MDCT technology and optimization of acquisition time in multiphasic imaging, still, pancreatic MDCT imaging is faced by a number of challenging imaging scenarios, such as identifying small early-stage hypo/isoatttenuating pancreatic adenocarcinoma and subtle enhancing components of cystic lesions that point to malignancy $[8,9]$. This results in either missed or equivocal lesions with subsequent recall of patients for additional imaging studies, such as MRI, EUS, and PET/CT, which may increase radiation exposure and patient's anxiety as well as treatment cost [10-12].

Challenges facing CT diagnostics could be primarily attributed to the limited contrast between the lesions and surrounding pancreatic parenchyma on CT. Therefore, if the contrast between pancreatic lesions and normal pancreatic parenchyma is improved on CT images, we may expect improved CT detectability of pancreatic lesions $[8,9]$.

Over the past several years, efforts have been directed to utilizing dual-energy CT (DECT) as an optimal advance of CT technology to improve pancreatic tumor detectability [13-16]. DECT uses two different x-ray energy peaks to acquire images. Since DECT exploits varying $\mathrm{x}$-ray attenuation properties of different materials and tissues at different energies; therefore, tissues having similar densities but different elemental compositions can be differentiated [17].

DECT scanners have been marketed with different technologies, based mainly on modifications of the X-ray tube output (e.g., two X-ray tubes with different peak energies-dual-source $\mathrm{CT}$-or single tube with rapid switching between high- and low-energy levels-rapidKVp switching CT) $[18,19]$.

Dual-layer CT (DLCT), on the other hand, is the first DECT machine based on the separation of high- and low-energy photons at the detector level obtaining both conventional images and retrospectively available spectral data with every scan without the need for prescan decision making, unlike tube-based DECT scanners. This data can be used to generate a variety of spectral image series, among which are virtual monoenergetic (VME) images [20].

VME images simulate datasets that would result if images were acquired using a true single-energy $x$-ray beam and can be calculated as linear combinations of the two datasets (high- and low-energy data) [21]. This is obtained across a wide range of energies (measured in kiloelectron volts, $\mathrm{keV}$ ), with a lower limit exceeding iodine k-edge $(33 \mathrm{keV})[22,23]$. Owing to the higher attenuation of low-energy X-ray photons, low-energy VME images result in greater soft-tissue contrast resolution and on administration of iodinated contrast agent, in boosted visualization of tissues that have taken up iodinated contrast compared to the surrounding tissues, due to increased photoelectric effect at energies in the proximity of the k-edge of iodine [24, 25].

The current study attempted to evaluate the detectability of hypoattenuating focal pancreatic lesions using virtual monoenergetic reconstructions generated from contrast-enhanced dual-layer CT in comparison to conventional polyenergetic images.

\section{Methods}

\section{Patient population}

Forty-seven consecutive patients were retrospectively identified using the radiologic information system and were included in this study according to the following inclusion criteria: (a) underwent a dual-layer CT of the abdomen between July 2016 to February 2017, comprising at least a portal venous phase, (b) had at least one definite or suspected hypoattenuating pancreatic lesion mentioned in their radiology report, and (c) had age above 18 years. The institutional review board approved this study and written informed consent was waived due to the retrospective design of the study. All the CT scans performed were clinically indicated for various clinical scenarios. None of the scans were performed solely for the purpose of this study.

\section{Image acquisition}

All patients were imaged using dual-layer CT (IQon, Philips Healthcare, Amsterdam, the Netherlands). The patients were imaged supine, craniocaudally during inspiration with breath hold. Intravenous iodinated contrast medium (Accupaque-350 ${ }^{\circ}$, GE-Healthcare) was administered to all patients with a body-weight adapted volume, power-injected at a mean rate of 3.5 $\mathrm{ml} / \mathrm{s}$ followed by a $30-\mathrm{ml}$ normal saline chaser. Every patient was imaged following the appropriate scan protocol based on the clinical request, for example, 
search for clinically suspected pancreatic cancer, staging of known pancreatic cancer, metastatic workup for a known intra/extra-abdominal primary malignancy, i.e., not all the patients had undergone a dedicated multiphasic pancreatic protocol. Portal venous phase was the common phase acquired for all the patients. All portal venous scans were initiated with a 50-s delay after reaching a threshold of $150 \mathrm{HU}$ in the abdominal aorta (bolus tracking). Tube current was automatically modulated according to patient's size. The following parameters were applied: tube voltage $120 \mathrm{kVp}$, collimation $64 \times 0.625 \mathrm{~mm}$, tube rotation time $0.5 \mathrm{~s}$, pitch 0.671 , and mean CTDI 10.5 .

\section{Image reconstruction and post-processing}

Reconstruction of conventional polyenergetic images and VME images $(40-200 \mathrm{KeV})$ was performed using a dedicated workstation (Intellispace Portal 9.0, Philips Healthcare, The Netherlands) at 2-mm slice thickness. Conventional images were reconstructed using iterative reconstruction (iDose ${ }^{4}$, strength level 3, Philips Healthcare, the Netherlands), as performed in clinical routine. VME images $(40-200 \mathrm{KeV})$ were reconstructed using a dedicated spectral reconstruction algorithm (Spectral B, strength level 3, Philips Healthcare, the Netherlands). All further analyses were done using the same dedicated workstation. Only portal venous phase images were analyzed. Analysis was performed by a radiologist with 7 years of experience, blinded to all clinical data, radiologic report, pathology, and final diagnosis.

\section{Quantitative analysis}

Circular regions of interest (ROIs) were placed in the following regions: identified lesion, normal pancreatic parenchyma, and retroperitoneal fat. ROIs were placed in conventional images at first and then copied to VME images (40 KeV to $200 \mathrm{KeV}$ with $10 \mathrm{KeV}$ intervals). When more than one lesion was identified, a ROI was drawn for every lesion. Also in case of complex cystic lesions with identified solid component, a ROI was placed on every component (solid and cystic) and analyzed separately. The ROI's size and location were identical in all image sets, avoiding margins, calcifications, vessels, and necrotic areas. Each ROI was measured 3 times and the mean Hounsfield unit value and standard deviation (SD) was recorded. Contrast-to-noise ratio (CNR) for conventional images and VME image sets $(40-200 \mathrm{KeV}, 10-\mathrm{KeV}$ interval) was calculated as difference between ROI value of normal enhancing pancreas and pancreatic lesion and divided by the SD of a homogenous portion of retroperitoneal fat as background noise. $\mathrm{CNR}=(\mathrm{ROI}$ pancreas - ROI lesion)/SD fat. Signal-to-noise ratio (SNR) is calculated as a ratio of the ROI value of the observed lesion to SD of homogenous portion of retroperitoneal fat.

\section{Qualitative analysis}

Subjectively, conventional reconstructions and two selected low-level VME reconstructions (40 and $50 \mathrm{KeV}$ ) were evaluated regarding lesion visibility using a 4-point scale: 1 , poorly visible, to 4 , prominently visible. The different types of image reconstructions were randomized in the research folders to prevent lesion recall, since every lesion was evaluated 3 times in 3 image sets. Also the reader was blinded to type of image reconstruction. Optimizing window settings of different image sets for optimal lesion visualization was allowed.

\section{Lesion validation}

Validation of the identified lesions was based on histopathologic confirmation whenever possible. Lesions that warranted conservative management by follow-up were verified by a combination of typical imaging features seen on additional imaging studies that the patients had undergone according to their management plan, including follow-up CT, MRCP, and/or EUS along with clinical data.

\section{Statistical analysis}

All statistical analysis was performed using IBM SPSS software package version 20.0. (Armonk, NY: IBM Corp). Wilcoxon signed rank test was used to determine the significance of differences in CNR in conventional images compared to various $\mathrm{KeV}$ level VME images and between different levels VME images. The recorded subjective image ranks were tested for statistical significance using Wilcoxon test. The number and percent of each rank in various image sets was also recorded. A $p$ value $<0.05$ was considered statistically significant.

\section{Results}

The study included 47 patients, 29 males (62\%) and 18 females (38\%) with mean age 66 years (age range $34-$ 92 years). All had focal pancreatic lesions. Six patients had more than one focal lesion. One patient had a complex cystic lesion with a suspected solid component, which was analyzed as two separate lesions. A total of 54 lesions were analyzed; pancreatic adenocarcinoma $(n=14)$, metastases (from renal cell carcinoma, malignant melanoma, ovarian, and colorectal cancer) $(n=4)$, neuroendocrine tumors $(n=3)$, lymphoma $(n=2)$, chronic mass-forming pancreatitis $(n=1)$, fibrosis (post-operative, post-irradiation) $(n=2)$, focal fatty infiltration $(n=1)$, mural nodule of cystic mucinous neoplasm $(n=1)$, mucinous cystic neoplasm $(n=1)$, pseudocyst $(n=6)$, and intraductal mucinous cystic neoplasm $(n=19)$. 
Sixteen of 54 lesions $(\approx 30 \%)$ were discovered incidentally in patients who were referred for $\mathrm{CT}$ for clinical indications unrelated to pancreatic disease.

Lesion validation was done either histopathologically ( $n=21$ lesions) or using EUS/MRCP $(n=9)$, follow-up CT (follow-up duration 2-6 months) $(n=26)$ combined with clinical data (two of the lesions were verified with more than one imaging modality). All pancreatic ductal adenocarcinomas and neuroendocrine tumors $(n=19)$ were verified by histopathology.

\section{Quantitative analysis}

In all 54 lesions, across the VME range, CNR and SNR showed gradual decline with increasing $\mathrm{KeV}$ level (Fig. 1). On $40 \mathrm{KeV}$ images, which is the lowest $\mathrm{KeV}$ level generated, CNR and SNR were highest compared to all $\mathrm{KeV}$ levels $(p<0.001, p<0.001)$ (Fig. 1, Tables 1 and 2). In comparison to conventional images, CNR and SNR were significantly higher on VME images at 40-80 KeV level (Tables 1 and 2).

Lower noise was seen at higher KeV level VME images compared to lower levels. Image noise was significantly highest at $40 \mathrm{KeV}(p<0.001)$ (Table 3$)$. Despite that, mean noise was significantly lower in all $\mathrm{KeV}$ levels including $40 \mathrm{KeV}$ in comparison to conventional images $(p<0.001)$ (Table 3).

\section{Qualitative analysis}

Regarding subjective evaluation of lesion visibility, the highest scores were observed on $40 \mathrm{KeV}$ followed by $50 \mathrm{KeV}$ reconstructions without a statistical significant difference between them $(4(2-4)$ vs $4(2-4)$, respectively, $p=0.317$ ), where $83.3 \%$ and $81.5 \%$ of lesions were considered prominently visible (score 4) on 40 $\mathrm{KeV}$ images and $50 \mathrm{KeV}$ images, respectively, and both were significantly higher when compared to conventional image set $(3(1-4), p<0.001, p<0.001)$ with only $35.2 \%$ of the lesions rated 4 on conventional images (Figs. 2 and 3).

\section{Discussion}

DECT has already shown increased capabilities in abdominal imaging over the last years [22, 26]. A number of earlier studies have also found DECT to be useful in the diagnostic evaluation of pancreatic lesions. The majority of these studies were performed using tube-based DECT systems [14, 15, 27, 28]. The present study investigated virtual monoenergetic images generated from a novel detector-based DECT

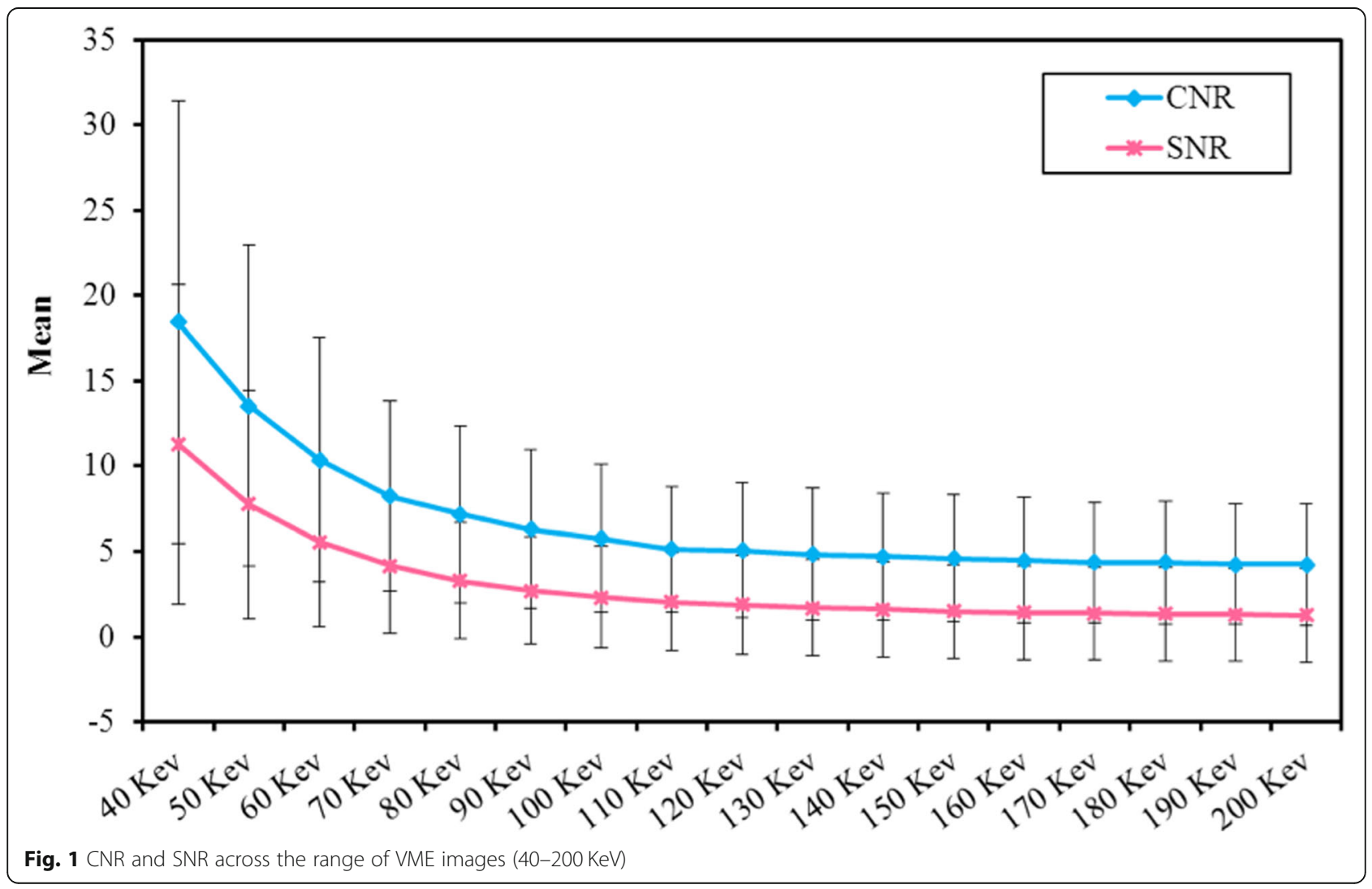


Table 1 Quantitative analysis of image sets (conventional images and VME images 40-120 KeV) according to CNR

\begin{tabular}{llll}
\hline & CNR & $\boldsymbol{p}_{\text {Conventional }}$ & $\boldsymbol{p}_{\mathbf{4 0 ~ K e V}}$ \\
\hline Conventional & $6.63 \pm 4.92$ & & \\
$40 \mathrm{KeV}$ & $18.47 \pm 12.98$ & $<0.001^{*}$ & \\
$50 \mathrm{KeV}$ & $13.55 \pm 9.41$ & $<0.001^{*}$ & $<0.001^{*}$ \\
$60 \mathrm{KeV}$ & $10.36 \pm 7.18$ & $<0.001^{*}$ & $<0.001^{*}$ \\
$70 \mathrm{KeV}$ & $8.24 \pm 5.56$ & $<0.001^{*}$ & $<0.001^{*}$ \\
$80 \mathrm{KeV}$ & $7.17 \pm 5.18$ & $0.004^{*}$ & $<0.001^{*}$ \\
$90 \mathrm{KeV}$ & $6.31 \pm 4.63$ & 0.051 & $<0.001^{*}$ \\
$100 \mathrm{KeV}$ & $5.76 \pm 4.33$ & $<0.001^{*}$ & $<0.001^{*}$ \\
$110 \mathrm{KeV}$ & $5.14 \pm 3.68$ & $<0.001^{*}$ & $<0.001^{*}$ \\
$120 \mathrm{KeV}$ & $5.07 \pm 3.97$ & $<0.001^{*}$ & $<0.001^{*}$ \\
${ }^{\mathrm{Fr}} \boldsymbol{p}$ & $<0.001^{*}$ & & \\
${ }^{*}$ statistically significant & &
\end{tabular}

(dual-layer CT), to determine whether these images can offer improved detectability of hypoattenuating focal pancreatic lesions over conventional images.

Improved CNR and SNR were found at low $\mathrm{KeV}$ VME images over higher $\mathrm{KeV}$ and conventional images. The highest values were achieved at $40 \mathrm{KeV}$, which is the lowest $\mathrm{KeV}$ level generated. This is attributed to the higher attenuation of tissues with iodinated contrast at low $\mathrm{KeV}$ monoE images close to the iodine $\mathrm{K}$ edge (33 $\mathrm{KeV})$. These findings were similar to findings reported in other studies using tube-based dual-energy CT [15, 29, 30]. Bhosale et al. [14] demonstrated that CNR for pancreatic ductal adenocarcinoma at low monochromatic energy images $(50-70 \mathrm{KeV})$ generated by a rapidKVp switching dual-energy CT is significantly higher compared to conventional polychromatic (120 KVp) images. Lin et al. [31], who studied hypervascular pancreatic neuroendocrine tumors also using a rapid-KVp

Table 2 Quantitative analysis of image sets (conventional images and VME images 40-120 KeV) according to SNR

\begin{tabular}{llll}
\hline & SNR & $p_{\text {Conventional }}$ & $\boldsymbol{p}_{\mathbf{4 0 ~ K e V}}$ \\
\hline Conventional & $2.83 \pm 3.21$ & & \\
$40 \mathrm{KeV}$ & $11.28 \pm 9.40$ & $<0.001^{*}$ & \\
$50 \mathrm{KeV}$ & $7.76 \pm 6.68$ & $<0.001^{*}$ & $<0.001^{*}$ \\
$60 \mathrm{KeV}$ & $5.55 \pm 4.93$ & $<0.001^{*}$ & $<0.001^{*}$ \\
$70 \mathrm{KeV}$ & $4.15 \pm 3.99$ & $<0.001^{*}$ & $<0.001^{*}$ \\
$80 \mathrm{KeV}$ & $3.28 \pm 3.42$ & $<0.001^{*}$ & $<0.001^{*}$ \\
$90 \mathrm{KeV}$ & $2.71 \pm 3.17$ & 0.284 & $<0.001^{*}$ \\
$100 \mathrm{KeV}$ & $2.32 \pm 3.0$ & $<0.001^{*}$ & $<0.001^{*}$ \\
$110 \mathrm{KeV}$ & $2.04 \pm 2.89$ & $<0.001^{*}$ & $<0.001^{*}$ \\
$120 \mathrm{KeV}$ & $1.89 \pm 2.91$ & $<0.001^{*}$ & $<0.001^{*}$ \\
${ }^{*} \boldsymbol{p}$ & $<0.001^{*}$ & & \\
\hline * statistically significant & &
\end{tabular}

Table 3 Quantitative analysis of image sets (conventional polyenergetic images and VME images 40-120 KeV) according to image noise

\begin{tabular}{llll}
\hline & Noise (SD) & $\boldsymbol{p}_{\text {Conventional }}$ & $\boldsymbol{p}_{\mathbf{4 0 ~ K e V ~}}$ \\
\hline Conventional & $12.99 \pm 4.72$ & & \\
$40 \mathrm{KeV}$ & $10.47 \pm 3.68$ & $<0.001^{*}$ & \\
$50 \mathrm{KeV}$ & $10.09 \pm 3.49$ & $<0.001^{*}$ & $<0.001^{*}$ \\
$60 \mathrm{KeV}$ & $9.93 \pm 3.39$ & $<0.001^{*}$ & $<0.001^{*}$ \\
$70 \mathrm{KeV}$ & $9.93 \pm 3.27$ & $<0.001^{*}$ & $<0.001^{*}$ \\
$80 \mathrm{KeV}$ & $9.84 \pm 3.28$ & $<0.001^{*}$ & $<0.001^{*}$ \\
$90 \mathrm{KeV}$ & $9.82 \pm 3.28$ & $<0.001^{*}$ & $<0.001^{*}$ \\
$100 \mathrm{KeV}$ & $9.78 \pm 3.27$ & $<0.001^{*}$ & $<0.001^{*}$ \\
$110 \mathrm{KeV}$ & $9.79 \pm 3.24$ & $<0.001^{*}$ & $<0.001^{*}$ \\
$120 \mathrm{KeV}$ & $9.77 \pm 3.27$ & $<0.001^{*}$ & $<0.001^{*}$ \\
${ }^{*} \boldsymbol{p r}$ & $<0.001^{*}$ & & \\
${ }^{*}$ statistically significant & &
\end{tabular}

switching dual-energy CT system, found that the best monoenergetic level based on CNR of pancreatic tumors is $45-60 \mathrm{KeV}$ range. Results by Hardie et al. [32] were also concordant with results reached in the present study, where CNR of pancreatic masses was significantly greater for $55 \mathrm{KeV}$ images obtained from a dual-source CT system compared to routine data set.

An interesting aspect in this study is the lower image noise observed at all $\mathrm{KeV}$ levels, even the lowest level (40 KeV) VME images, compared to conventional images. This result contrasted with results of other study groups $[32,33]$. This discrepancy could be traced back to the difference in dual-energy technologies used. Duallayer CT used in the present study, unlike other dualenergy technologies, allows reconstruction of VME images from fully matched low- and high-energy data in time and location at projection domain, permitting the suppression of anti-correlated noise between the two data sets [34]. Moreover, dual-layer CT allows the application of iterative reconstruction methods in spectral reconstruction algorithms, which leads to a further diminution in image noise [35]. Among the available tube-based dual-energy CT systems, only the rapid-kVp switching technology permits post-processing of raw data in projection domain to obtain VME datasets, but this is possible only after temporal and angular data interpolation, a process which results in accumulation of image noise from the high and low CT images of the independent scans [36, 37]. Other available tube-based scanners generate VME datasets in the image domain with significant increase of image noise at low-energy VME levels [24].

The results of subjective analysis were a clear reflection to the quantitative measurements, where subjective lesion visibility on lowest $\mathrm{KeV}$ VME images (40, $50 \mathrm{KeV}$ ) 


\section{Lesion visibility}

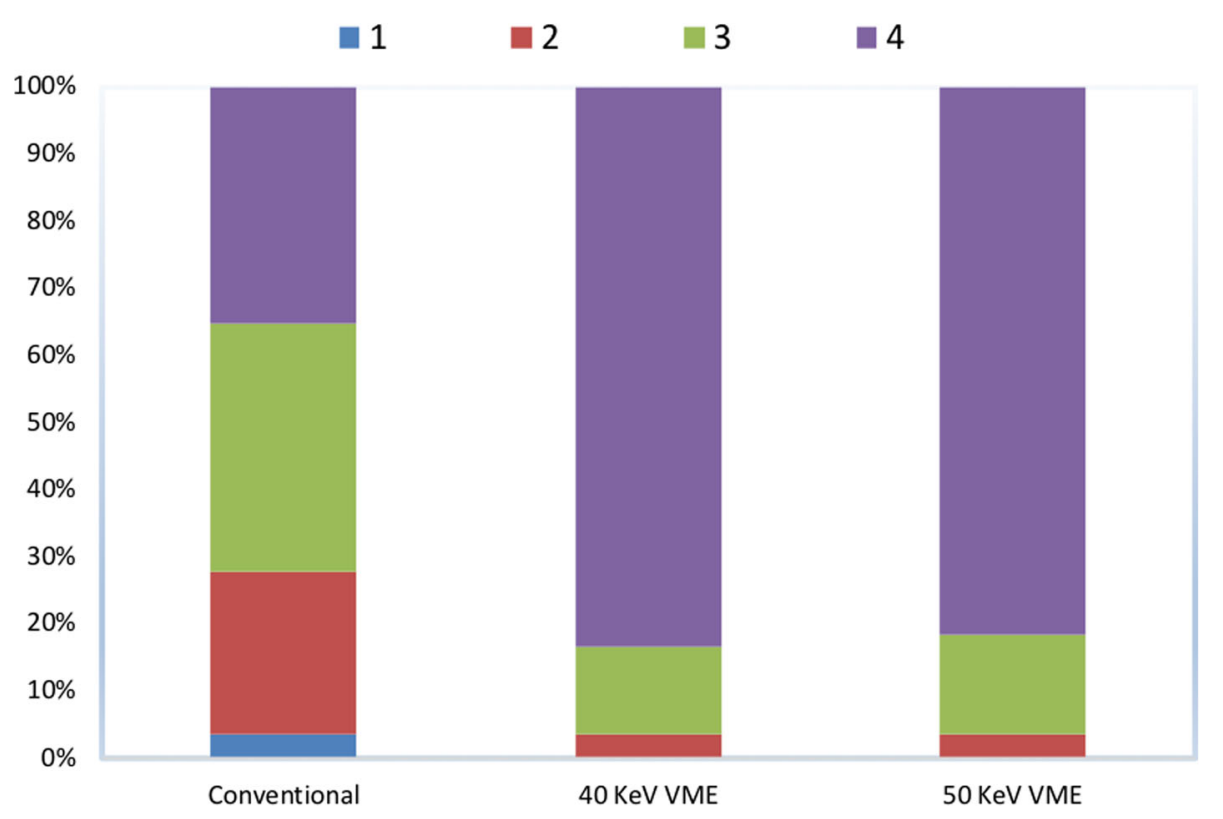

Fig. 2 Qualitative analysis of image sets (40 KeV, $50 \mathrm{KeV}$, and conventional) according to lesion visibility

significantly surpassed that of conventional images, mostly as a result of significant increase in lesion to parenchyma contrast coupled with low noise on these image sets.

In the current study, lesion verification was done by pathology for all lesions that warranted biopsy or surgical resection such as pancreatic adenocarcinomas or neuroendocrine tumors. Pathologically unverified lesions were those lesions indicated for conservative management and imaging follow-up, such as incidental $<2 \mathrm{~cm}$ IPMNs followed by CT, pancreatic metastases/lymphoma with widespread malignancy followed by CT after chemo/radiotherapy, post-operative fibrosis showing regression on close follow-up CT, and pseudocysts in patients with history of recent pancreatitis. This followup CT verified the presence of these lesions and supported their diagnosis. Knowing that the prime focus of this study was to evaluate detectability of the lesions by DLCT VME images, study of pathologies by VME images was out of the scope of this study.

Despite that the entire analysis was done in the portal venous phase, the current study revealed that low-energy VME images $(40,50 \mathrm{KeV})$ generated from a single venous phase scan of dual-layer CT were by far superior to venous phase conventional CT images. Low KeV VME images may offer advantages in detecting small lesions or lesions having minimal contrast to the adjacent pancreatic tissue. Accordingly, in certain selected cases with incidentally detected pancreas lesions, it might be possible to disregard additional dedicated pancreatic imaging protocols-with subsequent reduction of radiation exposure, if lesion visibility was high enough using low VME images of the first routine scan acquired in portal phase; however, this has to be further validated in future research.

This study had some limitations. First, patient selection for the study might form a limitation as it depended on CT reports that mentioned hypoattenuating pancreatic lesions, so that only patients were included with pancreatic lesions that were already suspected or detected on conventional images. Thus, results might not represent some very low-contrast or extremely small-size lesions. In the future, routine assessment of low $\mathrm{KeV}$ reconstructions for every scan may be useful for validating the value of VME imaging for such lesions. Second, the current study results reflected a primary single-center experience with a small number of patients that presented with a diversity of pancreatic pathologies. These results should be further validated in future larger-scale studies with more homogenous cohorts of lesions. Last is several lesions were not pathologically verified; those were ones not indicated for biopsy or surgical resection based on the patient's management plan.

\section{Conclusion}

In conclusion, based on the qualitative analysis of this study and substantiated by quantitative measurements, dual-layer CT is considered a superior imaging choice 


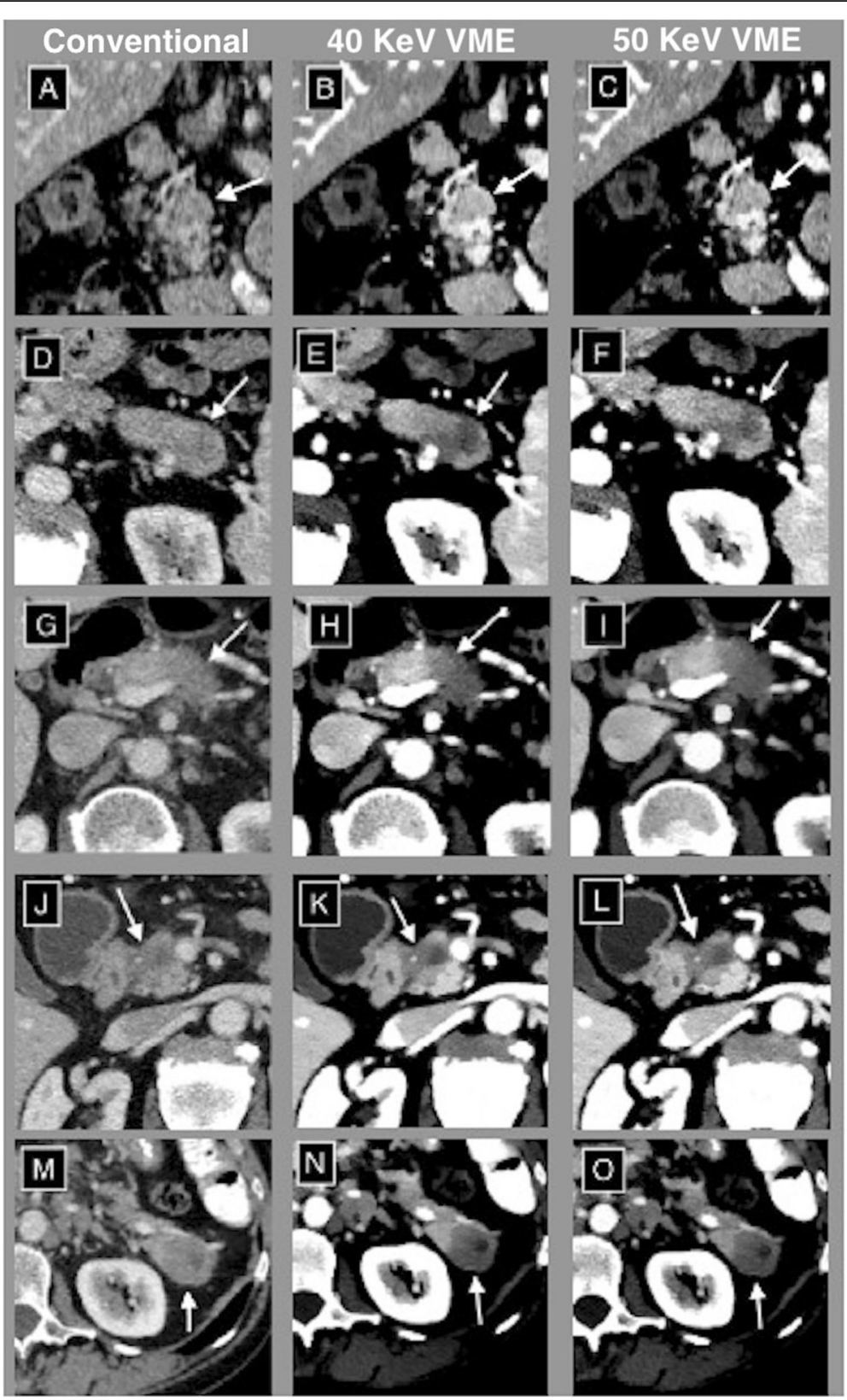

Fig. 3 Examples of conventional images (left column), $40 \mathrm{KeV}$ VME images (middle column) and $50 \mathrm{KeV}$ VME images (right column). Arrows demonstrate boosted visibility of pancreatic lesions in 40 and $50 \mathrm{KeV}$ VME images, showing more distinct boundaries and internal details (a-c hypovascular neuroendocrine tumor in the pancreatic head; $\mathbf{d}$, e hypovascular neuroendocrine tumor in the pancreatic tail; $\mathbf{g}$-i pancreatic ductal adenocarcinoma in the pancreatic body; $\mathbf{j}-\mathbf{I}$ uncinate process pancreatic adenocarcinoma showing internal necrosis and fully encased gastroduodenal artery; $\mathbf{m}, \mathbf{n}$ known metastasis from ovarian cancer with focal necrotic)

over conventional CT for detecting hypoattenuating pancreatic lesions. Adding low $\mathrm{KeV}(40$ and $50 \mathrm{KeV}$ ) VME reconstructions to the routine clinical pancreatic imaging-where possible-can boost detection of hypoattenuating focal pancreatic lesions including pancreatic adenocarcinoma, thus potentially improving prognosis of this fatal disease. Moreover, improved lesion detectability by DLCT could potentially restrict the need for additional imaging studies, thus reducing costs and radiation exposure.

\section{Abbreviations}

CNR: Contrast-to-noise ratio; DECT: Dual-energy computed tomography; DLCT: Dual-layer computed tomography; EUS: Endoscopic ultrasound; MDCT: Multidetector computed tomography; MRCP: Magnetic resonance 
cholangiopancreatography; MRI: Magnetic resonance imaging; PET/ CT: Positron emission tomography/computed tomography; PPP: Pancreatic parenchymal phase; PVP: Portal venous phase; ROI: Region of interest; SD: Standard deviation; SNR: Signal-to-noise ratio; VME: Virtual monoenergetic

\section{Acknowledgements}

Not applicable.

\section{Authors' contributions}

NE designed the study; acquired, analyzed and interpreted data; performed statistical analysis; and drafted the manuscript. DE revised and edited the manuscript. AM, DM, and ME reviewed the manuscript. All authors read and approved the manuscript.

\section{Funding}

Not applicable

\section{Availability of data and materials}

The data sets used and/or analyzed during the current study are available from the corresponding author on responsible request.

\section{Ethics approval and consent to participate}

The Research Ethics Committee of Alexandria Faculty of Medicine approved this study and waived informed consent due to its retrospective nature (Ethics committee reference number 020937, IRB No. 00012098, FWA No. 00018699). All study procedures involving human participants were carried out in accordance with the ethical standards of Declaration of Helsinki.

\section{Consent for publication}

The Research Ethics Committee of Alexandria Faculty of Medicine approved this study and waived informed consent (Ethics committee reference number 020937, IRB No. 00012098, FWA No. 00018699).

\section{Competing interests}

David Maintz received speaker's honoraria from Philips Healthcare. For the remaining authors, no competing interests were declared.

Received: 4 June 2020 Accepted: 27 July 2020

Published online: 04 August 2020

\section{References}

1. Horton KM (2002) Multidetector CT and three-dimensional imaging of the pancreas: state of the art. J Gastrointest Surg 6:126-128

2. Bashir MR, Gupta RT (2012) MDCT evaluation of the pancreas: nuts and bolts. Radiol Clin N Am 50:365-377

3. Ros PR, Mortele KJ (2001) Imaging features of pancreatic neoplasms. JBRBTR 84:239-249

4. Sahani DV, Shah ZK, Catalano OA, Boland GW, Brugge WR (2008) Radiology of pancreatic adenocarcinoma: current status of imaging. J Gastroenterol Hepatol 23:23-33

5. Siegel RL, Miller KD, Jemal A (2019) Cancer statistics, 2019. CA Cancer J Clin 69:7-34

6. Callery MP, Chang KJ, Fishman EK, Talamonti MS, William Traverso L, Linehan DC (2009) Pretreatment assessment of resectable and borderline resectable pancreatic cancer: expert consensus statement. Ann Surg Oncol 16:1727-1733

7. Brennan DD, Zamboni GA, Raptopoulos VD, Kruskal JB (2007) Comprehensive preoperative assessment of pancreatic adenocarcinoma with 64-section volumetric CT. Radiographics 27:1653-1666

8. Chu AJ, Lee JM, Lee YJ, Moon SK, Han JK, Choi BI (2012) Dual-source, dualenergy multidetector $\mathrm{CT}$ for the evaluation of pancreatic tumours. $\mathrm{Br}$ J Radiol 85:e891-e898

9. De La Cruz MS, Young AP, Ruffin MT (2014) Diagnosis and management of pancreatic cancer. Am Fam Physician 89:626-632

10. Raman SP, Horton KM, Fishman EK (2012) Multimodality imaging of pancreatic cancer-computed tomography, magnetic resonance imaging, and positron emission tomography. Cancer J 18:511-522

11. Kim JH, Park SH, Yu ES, Kim MH, Kim J, Byun JH et al (2010) Visually isoattenuating pancreatic adenocarcinoma at dynamic-enhanced CT: frequency, clinical and pathologic characteristics, and diagnosis at imaging examinations. Radiology 257:87-96

12. Klapman J, Malafa MP (2008) Early detection of pancreatic cancer: why, who, and how to screen. Cancer Control 15:280-287

13. Johnson TR (2012) Dual-energy CT: general principles. AJR Am J Roentgenol 199:S3-S8

14. Bhosale P, Le O, Balachandran A, Fox P, Paulson E, Tamm E (2015) Quantitative and qualitative comparison of single-source dual-energy computed tomography and $120-\mathrm{kVp}$ computed tomography for the assessment of pancreatic ductal adenocarcinoma. J Comput Assist Tomogr 39:907-913

15. Patel BN, Thomas JV, Lockhart ME, Berland LL, Morgan DE (2013) Singlesource dual-energy spectral multidetector $C T$ of pancreatic adenocarcinoma: optimization of energy level viewing significantly increases lesion contrast. Clin Radiol 68:148-154

16. Granata V, Fusco R, Catalano O, Setola SV, de Lutio di Castelguidone E, Piccirillo $\mathrm{M}$ et al (2016) Multidetector computer tomography in the pancreatic adenocarcinoma assessment: an update. Infect Agent Cancer 11:57

17. Almeida IP, Schyns LE, Ollers MC, van Elmpt W, Parodi K, Landry G et al (2017) Dual-energy CT quantitative imaging: a comparison study between twin-beam and dual-source CT scanners. Med Phys 44:171-179

18. Aran S, Shaqdan KW, Abujudeh HH (2014) Dual-energy computed tomography (DECT) in emergency radiology: basic principles, techniques, and limitations. Emerg Radiol 21:391-405

19. Goo HW, Goo JM (2017) Dual-energy CT: new horizon in medical imaging. Korean J Radiol 18:555-569

20. Gabbai M, Leichter I, Romman Z, Altman A, Sosna J (2013) The clinical impact of retrospective analysis in spectral detector dual energy body CT. Paper presented at radiological society of North America 2013 scientific assembly and annual meeting, McCormick Place, Chicago, 1- 6 December 2013.

21. Alvarez RE, Macovski A (1976) Energy-selective reconstructions in X-ray computerized tomography. Phys Med Biol 21:733-744

22. Marin D, Boll DT, Mileto A, Nelson RC (2014) State of the art: dual-energy CT of the abdomen. Radiology 271:327-342

23. Silva AC, Morse BG, Hara AK, Paden RG, Hongo N, Pavlicek W (2011) Dualenergy (spectral) CT: applications in abdominal imaging. Radiographics 31: 1031-1046 discussion 1047-1050

24. Yu L, Leng S, McCollough CH (2012) Dual-energy CT-based monochromatic imaging. AJR Am J Roentgenol 199:S9-S15

25. Apfaltrer P, Sudarski S, Schneider D, Nance JW, Haubenreisser H, Fink C et al (2014) Value of monoenergetic low-kV dual energy CT datasets for improved image quality of CT pulmonary angiography. Eur J Radiol 83: $322-328$

26. Agrawal MD, Pinho DF, Kulkarni NM, Hahn PF, Guimaraes AR, Sahani DV (2014) Oncologic applications of dual-energy CT in the abdomen. Radiographics 34:589-612

27. Di Maso LD, Huang J, Bassetti MF, DeWerd LA, Miller JR (2018) Investigating a novel split-filter dual-energy $C T$ technique for improving pancreas tumor visibility for radiation therapy. J Appl Clin Med Phys 19: 676-683

28. McNamara MM, Little MD, Alexander LF, Carroll LV, Beasley TM, Morgan DE (2015) Multireader evaluation of lesion conspicuity in small pancreatic adenocarcinomas: complimentary value of iodine material density and low keV simulated monoenergetic images using multiphasic rapid kVpswitching dual energy CT. Abdom Imaging 40:1230-1240

29. Brooks RA (1977) A quantitative theory of the Hounsfield unit and its application to dual energy scanning. J Comput Assist Tomogr 1:487-493

30. Macari M, Spieler B, Kim D, Graser A, Megibow AJ, Babb J et al (2010) Dualsource dual-energy MDCT of pancreatic adenocarcinoma: initial observations with data generated at $80 \mathrm{kVp}$ and at simulated weightedaverage $120 \mathrm{kVp}$. AJR Am J Roentgenol 194:W27-W32

31. Lin XZ, Wu ZY, Tao R, Guo Y, Li JY, Zhang J et al (2012) Dual energy spectral CT imaging of insulinoma-value in preoperative diagnosis compared with conventional multi-detector CT. Eur J Radiol 81:2487-2494

32. Hardie AD, Picard MM, Camp ER, Perry JD, Suranyi P, De Cecco CN et al (2015) Application of an advanced image-based virtual monoenergetic reconstruction of dual source dual-energy $C T$ data at low keV increases image quality for routine pancreas imaging. J Comput Assist Tomogr 39: $716-720$ 
33. Albrecht MH, Scholtz JE, Husers K, Beeres M, Bucher AM, Kaup M et al (2016) Advanced image-based virtual monoenergetic dual-energy CT angiography of the abdomen: optimization of kiloelectron volt settings to improve image contrast. Eur Radiol 26:1863-1870

34. Kalender WA, Klotz E, Kostaridou L (1988) An algorithm for noise suppression in dual energy CT material density images. IEEE Trans Med Imaging 7:218-224

35. Chang W, Lee JM, Lee K, Yoon JH, Yu MH, Han JK et al (2013) Assessment of a model-based, iterative reconstruction algorithm (MBIR) regarding image quality and dose reduction in liver computed tomography. Investig Radiol 48:598-606

36. Dong X, Niu T, Zhu L (2014) Combined iterative reconstruction and imagedomain decomposition for dual energy $\mathrm{CT}$ using total-variation regularization. Med Phys 41:051909

37. Lehmann L, Alvarez R, Macovski A, Brody W, Pelc N, Riederer SJ et al (1981) Generalized image combinations in dual KVP digital radiography. Med Phys 8:659-667

\section{Publisher's Note}

Springer Nature remains neutral with regard to jurisdictional claims in published maps and institutional affiliations.

\section{Submit your manuscript to a SpringerOpen ${ }^{\circ}$ journal and benefit from:}

- Convenient online submission

- Rigorous peer review

- Open access: articles freely available online

High visibility within the field

- Retaining the copyright to your article

Submit your next manuscript at $\boldsymbol{\nabla}$ springeropen.com 